\title{
Variabilidade espacial de propriedades hidrodinâmicas de um Neossolo Regolítico sob pastagem e caatinga
}

\author{
José A. S. de Oliveira Júnior ${ }^{1}$, Eduardo S. de Souza ${ }^{2}$, Marcelo M. Correa ${ }^{3}$, \\ José R. de S. Lima ${ }^{4}$, Rodolfo M. S. Souza ${ }^{5}$ \& Luiz A. da Silva Filho ${ }^{6}$ \\ ${ }^{1}$ Unidade Acadêmica de Garanhuns/UFRPE. Garanhuns, PE. E-mail: aildosabino@hotmail.com (Autor correspondente) \\ ${ }^{2}$ Unidade Acadêmica de Serra Talhada/UFRPE. Serra Talhada, PE. E-mail: eduardosouza@uast.ufrpe.br \\ ${ }^{3}$ Unidade Acadêmica de Garanhuns/UFRPE. Garanhuns, PE. E-mail: marcelometri@yahoo.com \\ ${ }^{4}$ Unidade Acadêmica de Garanhuns/UFRPE. Garanhuns, PE. E-mail: romualdo@uag.ufrpe.br \\ ${ }^{5}$ Unidade Acadêmica de Serra Talhada/UFRPE. Serra Talhada, PE. E-mail: rodolfomssouza@gmail.com \\ ${ }^{6}$ Unidade Acadêmica de Garanhuns/UFRPE. Garanhuns, PE. E-mail: luiz_ap7@hotmail.com
}

Palavras-chave: manejo do solo

Beerkan

geoestatística

\begin{abstract}
R E S U M O
As propriedades hidrodinâmicas dos solos de pastagem e de caatinga, são afetadas por diversos fatores, sobretudo associados a aspectos espaciais e temporais relacionados às diferentes características dos sistemas radiculares e das taxas de pisoteio do gado, mas poucos resultados que avaliem o efeito da magnitude dessas fontes de variabilidade sobre as propriedades hidrodinâmicas dos solos têm sido relatados na literatura. Objetivou-se analisar a variabilidade espacial dos parâmetros que descrevem as curvas de retenção (van Genuchten) e da condutividade hidráulica (Brooks \& Corey) da água do solo, obtidos com a metodologia denominada "Beerkan", que se baseia em ensaios de infiltração simplificados e nas informações texturais do solo. Para isto foram realizadas medidas de infiltração e coletadas amostras de solo em duas áreas: uma área de pastagem (Brachiaria decumbens) e outra de caatinga. Os resultados mostraram que o uso do solo com pastagem alterou significativamente as propriedades analisadas quando comparadas com as do solo com caatinga, com alterações mais expressivas na densidade do solo e na condutividade hidráulica. A análise geoestatística identificou a existência de dependência espacial de alguns atributos estudados, permitindo o mapeamento das áreas experimentais.
\end{abstract}

Key words: soil management

Beerkan

geostatistic

\section{Spatial variability of hydrodynamic properties of a Regolithic Neosoil under pasture and 'caatinga'}

\begin{abstract}
A B S T R A C T
The hydrodynamic properties of soils under pasture and 'caatinga' (native dry forest) are affected by several factors, mainly associated with spatial and temporal aspects, due to differing root characteristics or livestock trampling rates, but few results that evaluate the effect of the magnitude of these sources of variability in the hydrodynamic properties of soils have been reported in the literature. The objective of this study was to analyse the spatial variability of parameters of water retention (van Genuchten) and hydraulic conductivity (Brooks \& Corey) curves, obtained with the "Beerkan" methodology based on simple infiltration experiments and soil texture data. These measures were obtained for two sampled areas: an area of pasture (Brachiaria decumbens) and another one of 'caatinga'. The results showed that the use of pasture altered significantly the analysed properties of soil when compared to the ones with the soil under 'caatinga', and that the most significant changes were found in soil density and hydraulic conductivity. The geostatistic analysis identified the existence of spatial dependency of the studied attributes allowing the mapping of experimental areas.
\end{abstract}

\section{INTRODUÇÃO}

O cultivo dos solos acarreta modificações em seus atributos dependendo da intensidade de preparo e do manejo utilizado. A degradação do solo, decorrente da compactação, destruição da estrutura e da degradação da matéria orgânica, provoca efeitos negativos nas propriedades físicas, hídricas, químicas e biológicas, com prejuízos ao desenvolvimento de plantas afetando, em consequência, a produtividade desses solos (Silva \& Cabeda, 2006).
Quando o solo é utilizado com pastagem o pisoteio intenso de animais ocasiona a compactação o que, por sua vez, contribui para uma redução severa na macroporosidade, aumento da densidade do solo e redução da infiltração de água nas camadas mais superficiais do solo. Essas alterações dependem sobremaneira do tipo de solo, da textura e do teor de matéria orgânica sendo mais acentuadas em solos argilosos do que em solos arenosos (Flores, 2007).

Para a implantação de técnicas agrícolas favoráveis, além de se levar em consideração a produção da planta atual 
deve-se considerar também que os solos devem manter sua capacidade produtiva (Souza et al., 2008b). A compreensão da variabilidade espacial das propriedades hidrodinâmicas dos solos é fundamental para o estudo de diversos processos hidrológicos e ecológicos em ambientes semiáridos. A intensificação do uso de terras demarcadas pela agropecuária e o desmatamento, em virtude do aumento da população e, assim, a expansão das fronteiras agrícolas, têm feito com que esses ambientes e, consequentemente, seus solos, fiquem cada vez mais degradados.

Normalmente, para se realizar estudos e se fazer afirmações sobre as propriedades hidrodinâmicas do solo como a curva de retenção da água no solo $\theta(\mathrm{h})$ e a curva de condutividade hidráulica $K(\theta)$ no campo, é necessário coletar um grande número de informações. Para a caracterização hidrodinâmica dos solos isto implica em ensaios de campo e laboratoriais bastante dispendiosos que demandam um período prolongado de execução, ante o que alguns pesquisadores passaram a utilizar métodos indiretos que se baseiam em dados do solo prontamente disponíveis, usuais e de baixo custo, tais como a textura, a densidade, a porosidade e o teor de matéria orgânico, dentre outros (Minasny \& Mcbratney, 2002). Comumente, referidas relações são chamadas funções de pedotransferência, FPT.

Haverkamp et al. (1996) mostraram, baseados na teoria do escalonamento por intermédio da análise dinâmica aplicada à equação de fluxo não saturado, que a completa identificação dos fatores de escala da equação do fluxo não saturado pode ser efetuada independente da equação de infiltração considerada e das condições de fronteiras impostas. Este trabalho deu origem à metodologia denominada "Beerkan" (Braud et al., 2004; Lassabatère et al., 2006; Souza et al., 2008a) que se baseia principalmente em ensaios simplificados de infiltração e na análise da distribuição dos tamanhos das partículas do solo.

Em comparação com outros métodos experimentais, o método Beerkan é eficiente, de baixo custo e apresenta grande vantagem, pois sua aplicação é bem mais simples e rápida. Tanto as FPT como o método Beerkan surgiram com a proposta de melhorar as perspectivas nas análises de variabilidade espacial das propriedades hidráulicas dos solos a partir das quantidades e qualidades nas estimativas dessas propriedades. Atualmente, já são encontrados, na literatura, alguns trabalhos que utilizaram a metodologia Beerkan para estudar a variabilidade espacial dos parâmetros de $\theta(h)$ e $K(\theta)$ (Souza et al., 2008b; Santos et al., 2012b; Furtunato et al., 2013).

Neste contexto buscou-se avaliar o impacto da pastagem nas propriedades hidrodinâmicas de um Neossolo Regolítico no Agreste de Pernambuco analisando a variabilidade espacial dos parâmetros das curvas $\theta(\mathrm{h})$ e $\mathrm{K}(\theta)$ descritas, respectivamente, pelos modelos de van Genuchten (1980) e de Brooks \& Corey (1964) obtidas a partir da metodologia denominada Beerkan, em duas áreas: uma área de pastagem (Brachiaria decumbens) e outra de mata nativa (caatinga).

\section{Material e Métodos}

O trabalho foi conduzido na fazenda Riacho do Papagaio no Município de São João - PE, cujas coordenadas geográficas são: $8^{\circ} 48^{\prime} 35^{\prime \prime}$ de latitude Sul e $36^{\circ} 24^{\prime} 20^{\prime \prime}$ ' de longitude Oeste e aproximadamente $690 \mathrm{~m}$ de altitude no período de agosto de 2011 a abril de 2012. As parcelas analisadas consistiram em 3,4 ha de solo com mata nativa (caatinga) e 4 ha de solo cultivado com pastagem (Brachiaria decumbens Stapf) (Figura 1). O solo da área em estudo é classificado como Neossolo Regolítico Eutrófico típico (Santos et al., 2012b); o relevo local é suave ondulado com declividade média de 2,5\%. O clima local é, segundo a classificação Köppen, do tipo BShs' ou seja, clima quente, seco, com chuvas de outono-inverno.

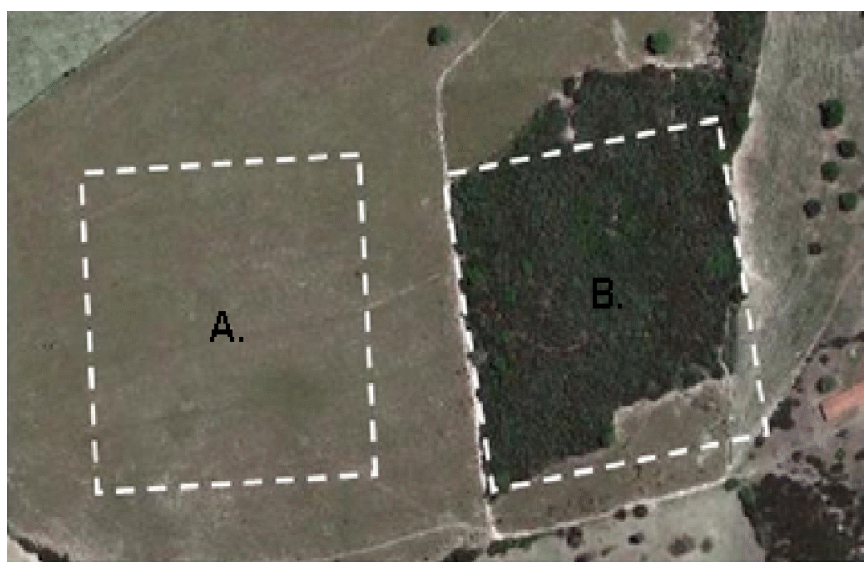

Figura 1. Imagem Google Earth evidenciando as duas áreas experimentais em que foram realizados os ensaios de infiltração e as coletas de solo, pastagem (A) e caatinga (B)

Os trabalhos experimentais mediram as propriedades hidrodinâmicas do solo em malhas de pontos equidistantes de 20 x 20 m sendo 121 pontos na parcela com pastagem e 101 pontos na parcela com caatinga; para isto foram realizados ensaios de infiltração no intuito de compor a análise do comportamento das propriedades hidrodinâmicas na pastagem e na mata nativa.

Os ensaios de infiltração foram realizados utilizando-se um infiltrômetro de anel simples com $150 \mathrm{~mm}$ de diâmetro, que consistem na anotação do tempo que volumes de água adicionados continuamente $(100 \mathrm{~mL})$ levam para infiltrar encerrando o ensaio quando o fluxo atinge o regime permanente, ou seja, de fluxo constante com o tempo. Durante os ensaios de infiltração foram coletadas amostras indeformadas visando à determinação da densidade do solo $\left(\rho_{s}\right)$ e amostras deformadas para determinação dos conteúdos volumétricos de água inicial $\left(\theta_{0}\right)$, final $\left(\theta_{s}\right)$ e para obtenção da curva granulométrica. A análise granulométrica foi realizada utilizando-se o método da ABNT (1984), o qual permite determinar os diâmetros das partículas mais finas (argila e silte) por sedimentação e as mais grosseiras (areia) por peneiramento.

\section{Metodologia Beerkan}

Nesta metodologia são determinados os parâmetros das curvas de retenção da água no solo $(\theta(\mathrm{h}))$ e de condutividade hidráulica do solo $(\mathrm{K}(\theta))$ descritas pelos modelos de van Genuchten (1980) e de Brooks \& Corey (1964) respectivamente: 


$$
\begin{gathered}
\left(\frac{\theta-\theta_{\mathrm{r}}}{\theta_{\mathrm{s}}-\theta_{\mathrm{r}}}\right)=\left[1+\left(\frac{\mathrm{h}}{\mathrm{h}_{\mathrm{g}}}\right)^{\mathrm{n}}\right]^{-\mathrm{m}} \operatorname{com} \mathrm{m}=1-\frac{2}{\mathrm{n}} \text { (Burdine, 1953) } \\
\mathrm{K}(\theta)=\mathrm{K}_{\mathrm{s}} \cdot\left(\frac{\theta-\theta_{\mathrm{r}}}{\theta_{\mathrm{s}}-\theta_{\mathrm{r}}}\right)^{\eta}
\end{gathered}
$$

sendo $\theta$ a umidade volumétrica $\left[\mathrm{L}^{3} \mathrm{~L}^{-3}\right] ; \theta_{\mathrm{r}}$ e $\theta_{\mathrm{s}}$ as umidades volumétricas residual e saturada $\left[\mathrm{L}^{3} \mathrm{~L}^{-3}\right]$, respectivamente; $h$ o potencial matricial $[\mathrm{L}] ; \mathrm{h}_{\mathrm{g}}[\mathrm{L}]$ um valor de escala de $\mathrm{h}$ considerado o potencial de entrada de ar; m e n são parâmetros de forma; $\mathrm{K}_{\mathrm{s}}$ a condutividade hidráulica saturada do solo [L $\mathrm{T}^{-1}$ ] e $\eta$ o parâmetro de forma para a curva de condutividade hidráulica.

A metodologia Beerkan se baseia nas propriedades estáticas e dinâmicas do solo, como a curva granulométrica e a curva de infiltração da água no solo, respectivamente para se obter os parâmetros $\left(\mathrm{m}, \mathrm{n}, \mathrm{\eta}, \mathrm{K}_{\mathrm{s}}, \mathrm{h}_{\mathrm{g}}\right)$.

Com vista à determinação dos parâmetros de forma $(\mathrm{m}, \mathrm{n}$ e $\eta$ ) e os de normalização $\left(K_{s}\right.$ e $\left.h_{g}\right)$ foi utilizado o BEST (Beerkan Estimation of Soil Transfer Parameters through Infiltration Experiments) (Lassabatére et al., 2006; Souza et al., 2008a).

Determinaram-se, através da estatística descritiva, medidas de posição: média aritmética e a mediana amostral e as medidas de dispersão, variância, desvio-padrão amostral e coeficiente de variação. A normalidade foi confirmada formalmente para todas as propriedades aplicando-se o teste de Kolmogorov-Smirnov com níveis de $95 \%$ ( $p>0,05)$ e $99 \%$ ( $p>0,01)$ de confiança. Determinou-se a diferença entre a frequência acumulada observada e a teórica máxima $\mid \mathrm{F}(\mathrm{O})$ - $\mathrm{F}(\mathrm{t}) \mid \max$, com base no número total de amostras da população observada dos dados experimentais e na distribuição teórica Normal. Comparando esta diferença com o valor admissível do coeficiente de Kolmogorov-Smirnov, conclui-se que a hipótese de normalidade não pode ser rejeitada se o valor $\mathrm{p}$ for maior ou igual a $\alpha$. A partir do coeficiente de variação o grau de variabilidade dos parâmetros foi analisado com base na classificação proposta por Warrick \& Nielsen (1980), que sugerem os limites CV < 12\%, 12 $<\mathrm{CV}<52 \%$ e $\mathrm{CV}>52 \%$ para as propriedades de baixa, média e alta variabilidade, respectivamente.

A metodologia utilizada para estudar a variabilidade espacial das propriedades hidrodinâmicas consiste em assimilar as $\mathrm{N}$ observações da variável considerada a uma realização bidimensional aplicando-se os princípios da análise geoestatística; o primeiro passo na análise geoestatística é a verificação da existência de dependência espacial que pode ser estimada pela Eq. 3 .

$$
\gamma(\mathrm{h})=\frac{1}{2 \mathrm{~N}(\mathrm{~h})} \sum_{\mathrm{i}=1}^{\mathrm{N}(\mathrm{h})}\left[\mathrm{Z}\left(\mathrm{x}_{\mathrm{i}}\right)-\left(\mathrm{x}_{\mathrm{i}}+\mathrm{h}\right)\right]^{2}
$$

sendo que:

$\gamma(\mathrm{h})$ - representa o valor do semivariograma estimado para a distância de separação (h) - entre as medidas

$\mathrm{Z}(\mathrm{xi})$ - se refere ao valor da variável regionalizada para a posição $x_{i}$ não estimado (verdadeiro)

$\mathrm{Z}\left(\mathrm{x}_{\mathrm{i}}+\mathrm{h}\right)$ - representa o valor da mesma variável na posição $\mathrm{x}_{\mathrm{i}}+\mathrm{h}$ em qualquer direção

h - distância de separação entre as medidas

$\mathrm{N}(\mathrm{h})$ - representa o número de pares experimentais de dados medidos de $\mathrm{Z}\left(\mathrm{x}_{\mathrm{i}}\right)$ e $\mathrm{Z}\left(\mathrm{x}_{\mathrm{i}}+\mathrm{h}\right)$

À análise e ao ajuste do semivariograma experimental a um modelo teórico denominam-se Análise Estrutural (Guerra, 1988). Esta estrutura é identificada por meio do estudo da forma do semivariograma $\gamma(\mathrm{h})$. Basicamente, a estrutura espacial é analisada dependendo do comportamento do semivariograma na origem e dos seus fenômenos de transição. De acordo com Carvalho et al. (2009), vários modelos teóricos de semivariogramas estão disponíveis na literatura, porém os que mais se destacam por permitir visualizar a natureza da variação espacial das variáveis que se pretende estudar, são o esférico Eq. (4), o exponencial Eq. (5) e o gaussiano Eq. (6):

$$
\begin{gathered}
\gamma(\mathrm{h})=\mathrm{C}_{0}+\mathrm{C}_{1}\left[\frac{3}{2}\left(\frac{\mathrm{h}}{\mathrm{A}}\right)-\frac{1}{2}\left(\frac{\mathrm{h}}{\mathrm{A}}\right)^{3}\right], 0<\mathrm{h}<\mathrm{A} \\
\gamma(\mathrm{h})=\mathrm{C}_{0}+\mathrm{C}_{1}\left[1-\mathrm{e}^{-\frac{\mathrm{h}}{\mathrm{A}}}\right], 0<\mathrm{h}<\mathrm{A} \\
\gamma(\mathrm{h})=\mathrm{C}_{0}+\mathrm{C}_{1}\left[1-\mathrm{e}^{-\frac{\mathrm{h}^{2}}{\mathrm{~A}^{2}}}\right], 0<\mathrm{h}<\mathrm{A} \\
\gamma(\mathrm{h})=\mathrm{C}_{0}+\mathrm{C}_{1}, \mathrm{~h} \geq \mathrm{A}_{0}
\end{gathered}
$$

sendo:

$$
\begin{aligned}
& \mathrm{C}_{0} \text { - efeito pepita } \\
& \mathrm{C}_{0}+\mathrm{C}_{1} \text { - patamar } \\
& \mathrm{A} \quad \text { - alcance }
\end{aligned}
$$

Para analisar o grau da dependência espacial dos atributosutilizou-se a classificação de Cambardella et al. (1994):

$$
\mathrm{GDE}=\frac{\mathrm{C}_{0}}{\mathrm{C}_{0}+\mathrm{C}_{1}}
$$

em que, são considerados de dependência espacial forte os semivariogramas que têm efeito pepita menor ou igual a $25 \%$ do patamar, moderada quando está entre 25 e $75 \%$ e fraca quando for maior que $75 \%$.

Constatando dependência ou correlação espacial, as estimativas para pontos não observados são obtidas pela técnica da Krigagem; trata-se de uma técnica de interpolação não tendenciosa que possui variância mínima cuja estimativa é obtida através do cálculo de uma média ponderada de um conjunto de observações ao redor de uma vizinhança, caso em que a estimativa associada à posição $\mathrm{x}_{0}$, pode ser obtida pela expressão: 


$$
\overline{\mathrm{Z}}\left(\mathrm{x}_{0}\right)=\sum_{\mathrm{i}=1}^{\mathrm{N}} \lambda_{\mathrm{i}} \mathrm{Z}\left(\mathrm{xi}_{\mathrm{i}}\right)
$$

sendo:

$\mathrm{Z}\left(\mathrm{x}_{0}\right)$ - valores estimados da variável em estudo

$\mathrm{x}_{0} \quad$ - ponto a ser estimado

$\mathrm{N}$ - número de vizinhos utilizado na estimativa

$\mathrm{Z}\left(\mathrm{x}_{0}\right) \lambda_{\mathrm{i}}$ - pesos ponderados associados a cada valor medido de $Z\left(x_{0}\right)$

A análise do comportamento visual dos semivariogramas é importante haja vista que fornece uma descrição sintética das estruturas dos fenômenos estudados. A adequação do modelo de semivariograma caracterizando a estrutura de um conjunto de pontos, deve ser verificada antes do mesmo ser utilizado para a geração de mapas, prognósticos e de cenários. O método de Jack - knifing, ou validação cruzada é aplicada usualmente para tal validação (Vieira et al., 2010); ele consiste em remover sucessivamente um ponto $Z_{i}$ de cada vez e depois estimá-lo a partir do restante de pontos produzindo um valor de $Z_{i}^{*}$; desta forma se definem, em cada localização dos pontos $\mathrm{X}_{\mathrm{i}}$, um erro reduzido $\mathrm{R}_{\mathrm{i}}$, seu valor médio e sua variância; o erro reduzido médio deve ser próximo de zero caso não exista erro sistemático e a variância deve ser próxima de um sugerindo que a variância dos erros $\left(Z_{i}-Z_{i}^{\star}\right)$ está consistente com a variância da krigagem $\left(\sigma_{\mathrm{KR}}\right)^{2}$.

Finalmente foram construídos, por meio da técnica de interpolação da Krigagem, os mapas de isolinhas de superfície representativos da distribuição espacial dos atributos hidrodinâmicos do solo. Os ajustes dos semivariograma foram realizados pelo método do quadrado mínimo ponderado e a escolha do melhor modelo foi baseada no Teste Jack-Knifing. Todas as análises geoestatística e a confecção dos mapas foram realizadas no R 3.0.2 (R Development Core Team, 2013) e o pacote geoR (Diggle \& Ribeiro Júnior, 2007).

Após a verificação da ausência de correlação a curta distância pelos semivariogramas das variáveis consideradas neste trabalho, a heterogeneidade espacial das variáveis foi caracterizada pela estatística clássica considerando que as observações são estatisticamente independentes umas das outras; neste caso, os mapas de isolinhas bidimensionais das variáveis não foram construídos.

\section{Resultados e Discussão}

A análise estatística das propriedades físico-hídricas, quais sejam: densidade do solo $\left(\rho_{\mathrm{s}}\right)$, umidade volumétrica inicial $\left(\theta_{0}\right)$ e umidade volumétrica final $\left(\theta_{\mathrm{s}}\right)$ é apresentada na Tabela 1 . Não foi observada diferença expressiva entre os valores médios de $\theta_{0}$ e de $\theta_{s}$ para as áreas com pastagem e caatinga; já para o valor de $\rho_{\mathrm{s}}$ foi observada pequena diferença significativa a $99 \%$ segundo o teste F; observa-se, também, maior amplitude nos valores de $\rho_{\mathrm{s}}$ para o solo com caatinga que para o solo com pastagem.

Com base na classificação proposta por Warrick \& Nielsen (1980) os parâmetros estatísticos da densidade do solo na pastagem e na caatinga indicaram baixa variabilidade nos dados com baixos coeficientes de variação ( $\mathrm{CV}<12 \%)$ (Tabela 1$)$. As alterações provocadas pelo pisoteio mais intenso do gado na pastagem evidenciam o aumento de $\rho_{\mathrm{s}}$, com menor amplitude e distribuição normal. A maior diversidade vegetal e o hábito dos animais formarem trilhas de caminhamento na caatinga fizeram com que a $\rho_{\mathrm{s}}$ apresentasse amplitude maior e seguisse uma distribuição lognormal (0,01 de nível significância). $\mathrm{O}$ incremento na $\rho_{\mathrm{s}}$ tem sido relatado por vários autores como o resultado da interferência do tráfego de máquinas e implementos agrícolas, do pisoteio de animais e da redução no teor de matéria orgânica em sistemas de pastagem (Oliveira et al., 2007; Severiano et al., 2009; Sales et al., 2010).

De acordo com os critérios estabelecidos por Warrick \& Nielsen (1980) as propriedades $\rho_{s}, \theta_{s}$ e $\theta_{0}$ são consideradas, respectivamente, como de baixa, média e alta variabilidade para as duas áreas analisadas.

Os resultados da análise geoestatística das propriedades hidrodinâmicas são apresentados na Tabela 2 .

A maioria dos semivariogramas experimentais foi ajustada pelo modelo esférico, $\operatorname{com}$ exceção do $\log \theta_{0}$ e do parâmetro $\mathrm{m}$, que se ajustaram aos modelos gaussiano e exponencial, respectivamente. A $\rho_{\mathrm{s}}\left(\mathrm{kg} \mathrm{dm}^{-3}\right)$ e $\theta_{\mathrm{s}}\left(\mathrm{cm}^{3} \mathrm{~cm}\right.$ ${ }^{3}$ ) na pastagem e a sorvidade na caatinga, apresentaram comportamento puramente aleatório. De conformidade com o critério estabelecido por Cambardella et al. (1994), todas as propriedades que apresentaram dependência espacial são consideradas moderadas com exceção de $m$ e $\eta$, ambos na pastagem, que foram forte e fraca, respectivamente.

Cambardella et al. (1994) estudando a variabilidade do solo em escala de campo associaram a dependência espacial forte com parâmetros intrínsecos do solo visto que estão relacionados

Tabela 1. Estatística das propriedades físico-hídricas dos solos sob pastagem e sob caatinga

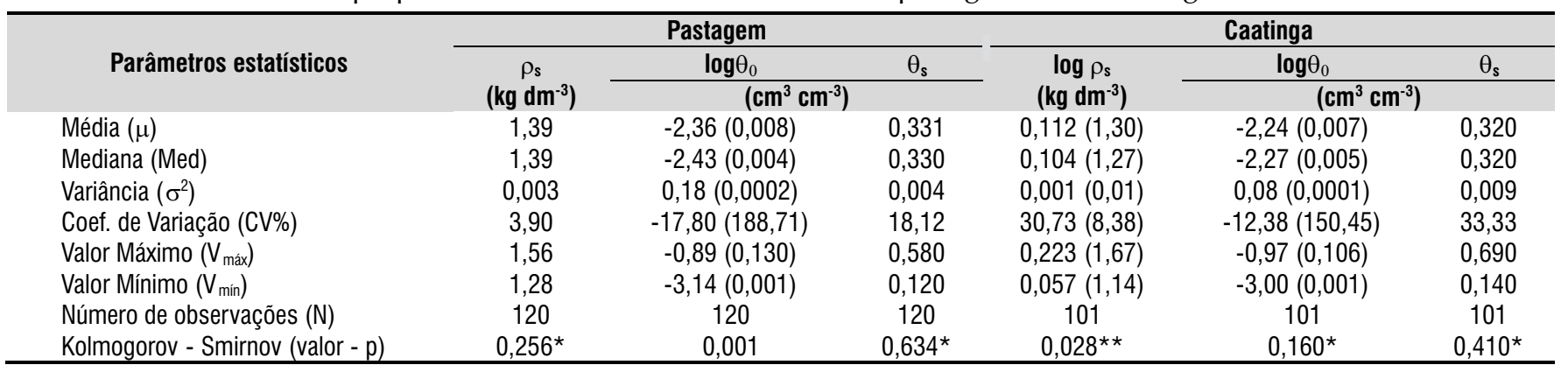

Entre parênteses são apresentados os valores sem transformação *Distribuição Normal a $95 \%$ de probabilidade $(p>0,05)$ **Distribuição Normal a $99 \%$ de probabilidade $(p>0,01)$ 
Tabela 2. Parâmetros da análise geoestatística das variáveis estudadas

\begin{tabular}{|c|c|c|c|c|c|c|c|}
\hline Propriedade & Modelo & CO & C & $A(m)$ & GDE (\%) & $\mu_{\mathrm{KR}}$ & $\left(\sigma_{K R}\right)^{2}$ \\
\hline$\rho_{s}\left(\mathrm{~kg} \mathrm{dm}^{-3}\right)$ & EPP & - & $\begin{array}{c}\text { Pastagem } \\
\text { - }\end{array}$ & . & , & r. & \\
\hline $\log \theta_{0}\left(\mathrm{~cm}^{3} \mathrm{~cm}^{-3}\right)$ & Gaussiano & 0,0880 & 0,0850 & 75,17 & 50,96 & 0,00122 & 0,9429 \\
\hline$\theta_{\mathrm{s}}\left(\mathrm{cm}^{3} \mathrm{~cm}^{-3}\right)^{\prime}$ & EPP & & & & & & \\
\hline $\mathrm{n}$ & Esférico & 0,0006 & 0,0004 & 57,78 & 60,00 & 0,0004 & 1,1595 \\
\hline $\mathrm{m}$ & Exponencial & 0,0000 & 0,0001 & 17,10 & 25,00 & 0,0004 & 1,2044 \\
\hline$\eta$ & Esférico & 0,3937 & 0,0810 & 82,71 & 82,94 & $-0,0003$ & 1,0734 \\
\hline $\mathrm{S}\left(\mathrm{mm} \mathrm{s}^{-1 / 2}\right)$ & Esférico & 0,0526 & 0,0298 & 39,86 & 63,83 & $-0,0001$ & 1,0075 \\
\hline $\mathrm{K}_{\mathrm{s}}\left(\mathrm{mm} \mathrm{s}^{-1}\right)^{\prime}$ & Esférico & 0,0011 & 0,0004 & 66,63 & 73,33 & $-0,0001$ & 0,9824 \\
\hline $\log \left|h_{g}\right|(\mathrm{mm})$ & Esférico & 0,0730 & 0,0327 & 68,42 & 69,06 & 0,00002 & 1,1169 \\
\hline & & & Caatinga & & & & \\
\hline $\log \rho_{\mathrm{s}}\left(\mathrm{kg} \mathrm{dm}^{-3}\right)$ & Esférico & 0,0007 & 0,0005 & 105,36 & 58,33 & 0,0016 & 1,0418 \\
\hline $\log \theta_{0}\left(\mathrm{~cm}^{3} \mathrm{~cm}^{-3}\right)$ & Esférico & 0,0400 & 0,0340 & 57,78 & 54,05 & $-0,0001$ & 1,1255 \\
\hline$\theta_{\mathrm{s}}\left(\mathrm{cm}^{3} \mathrm{~cm}^{-3}\right)$ & Esférico & 0,0060 & 0,0080 & 45,24 & 42,86 & 0,0026 & 0,9044 \\
\hline $\mathrm{m}$ & Esférico & 0,0007 & 0,0007 & 53,57 & 50,00 & $-0,0015$ & 0,9680 \\
\hline $\mathrm{n}$ & Esférico & 0,0063 & 0,0048 & 53,57 & 56,76 & $-0,0013$ & 0,9151 \\
\hline$\eta$ & Esférico & 1,2800 & 2,4500 & 44,96 & 34,32 & 0,0013 & 0,8732 \\
\hline $\mathrm{S}\left(\mathrm{mm} \mathrm{s}^{-1 / 2}\right)$ & EPP & - & - & - & - & - & - \\
\hline $\mathrm{K}_{\mathrm{s}}\left(\mathrm{mm} \mathrm{s}^{-1}\right)$ & Esférico & 0,0040 & 0,0042 & 35,38 & 48,78 & $-0,0013$ & 0,9504 \\
\hline $\log \left|h_{g}\right|(m m)$ & Exponencial & 0,0712 & 0,0563 & 63,98 & 55,84 & 0,0020 & 1,1183 \\
\hline
\end{tabular}

EPP - Efeito Pepita Puro

com a textura e com a mineralogia enquanto fatores extrínsecos, como cultivo e aplicação de fertilizantes, estão associados à dependência espacial fraca.

Os semivariogramas experimentais correspondentes às propriedades físico-hídricas $\left(\rho_{\mathrm{s}}, \log \theta_{0}\right.$ e $\left.\theta_{\mathrm{s}}\right)$ para os solos sob pastagem e sob caatinga são apresentados na Figura 2.

Nota-se que os semivariogramas experimentais das propriedades $\rho_{\mathrm{s}}$ e $\theta_{\mathrm{s}}$ do solo sob pastagem traduzem a inexistência de correlação espacial entre pontos amostrados indicando aspecto de pura aleatoriedade; assim, o efeito pepita poderia ser caracterizado melhor se medições com espaçamentos inferiores a $20 \mathrm{~m}$ tivessem sido realizadas.

A propriedade $\log \theta_{0}$ do solo sob pastagem e as propriedades $\rho_{\mathrm{s}}, \log \theta_{0}$ e $\theta_{\mathrm{s}}$ do solo sob caatinga, apresentaram dependência espacial sendo o modelo gaussiano ajustado ao primeiro e o esférico ajustado às demais propriedades (Figura 2). Apesar da densidade do solo ter apresentado distribuição lognormal, não foi necessário transformá-la para a análise do seu semivariograma.

$\mathrm{Na}$ Figura 3 são apresentados os mapas de isolinhas para as propriedade físico-hidrícas $\ln \theta_{0}$, na pastagem e $\rho_{s}, \log \theta_{0}$ e $\theta_{s}$ para a área de caatinga

Ao se analisar a distribuição espacial da caatinga nota-se que os maiores valores de $\rho_{\mathrm{s}}$ se encontram nas bordas da área estudada; esta distribuição espacial se deve ao fato dessa região ter maior probabilidade de sofrer interferência antrópica, como se pode observar pela presença de via de carro entre a pastagem e a caatinga (Figura 1B); outra questão é a possibilidade dos animais entrarem na vegetação em busca de sombra nos dias mais quentes.

A umidade inicial para as duas áreas é praticamente constante, com uma pequena variação no mapa da $\ln \theta_{0}$ do solo sob pastagem que em sua margem superior apresenta um aumento nos seus valores Uma vez que os ensaios foram realizados no período de agosto de 2011 a abril de 2012, uma
A.

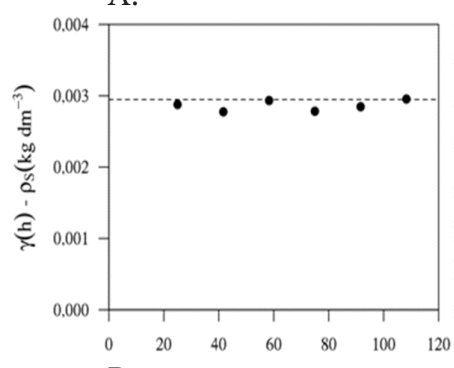

B.

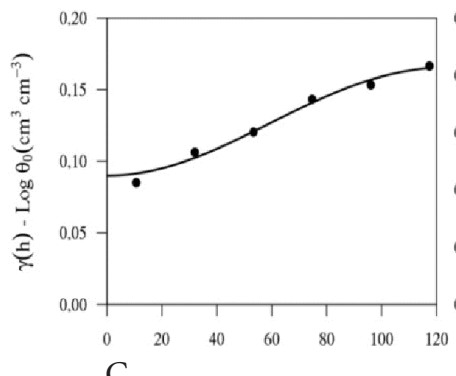

C.
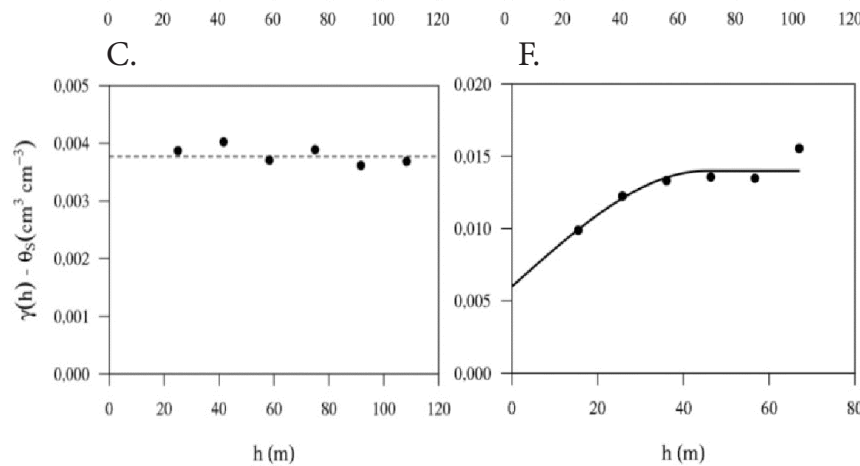
F.

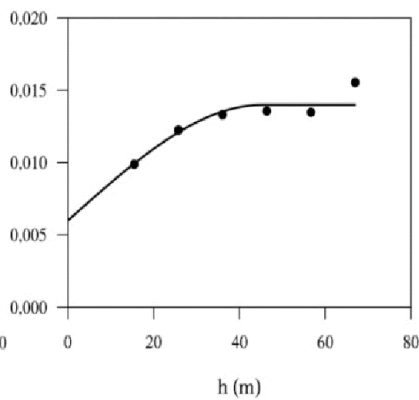

Figura 2. Semivariogramas experimental e teórico das propriedades físico-hídricas, $\rho_{s^{\prime}} \theta_{0}$ e $\theta_{\mathrm{s}}$ do solo sob pastagem (A, B e C) e sob caatinga ( $D, E$ e F)

variabilidade temporal deve ter afetado os valores da variável umidade volumétrica inicial visto que os ensaios não foram todos realizados no mesmo dia, ou seja, em período de mesmas condições climáticas. 


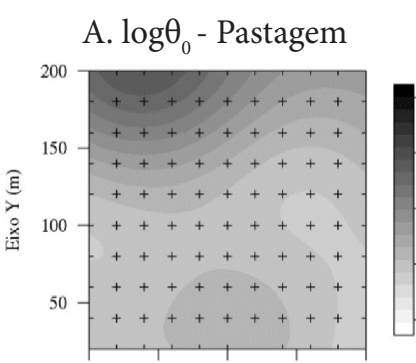

B. $\log \theta_{0}$ - Caatinga

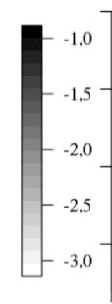

C. $\rho_{\mathrm{s}}$ - Caatinga
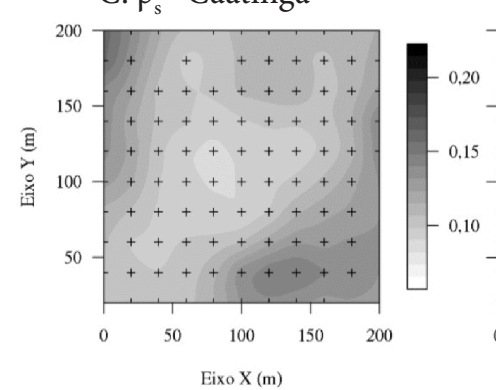

D. $\theta_{\text {s }}$-Caatinga

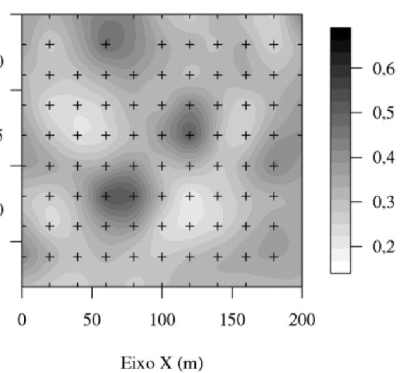

Figura 3. Mapa de isolinhas para as propriedades físico-hídricas, $\log \theta_{0}$ do solo sob pastagem e $\rho_{\mathrm{s},} \log \theta_{0}$ e $\theta_{\text {s }}$ sob caatinga

A estatística dos parâmetros $m$, $n$ e $\eta$ das curvas $\theta(h)$ e $K(\theta)$ (Eqs. 1 e 2) é apresentada na Tabela 3; os valores médios para m, n e $\eta$ para o solo sob pastagem são diferentes estatisticamente pelo teste $\mathrm{F}$ dos valores médios para o solo sob caatinga.

De acordo com os critérios de Warrick \& Nielsen (1980) os parâmetros de formas m e $n$ de $h(\theta)$ e $\eta$ de $K(\theta)$ para do solo sob pastagem apresentaram baixa e média variabilidade, respectivemente, enquanto que no solo sob caatinga foi obtida baixa variabilidade para n e média variabilidade para m e $\eta$ (Tabela 3). Os parâmetros de forma $m, n$ e $\eta$ da área de pastagem seguem distribuição normal segundo o teste Kolmogorov-Smirnov com $95 \%$ de probabilidade enquanto na área de caatinga, $\mathrm{m}$, segue uma distribuição normal com $99 \%$ de probabilidade, $\eta$ com $95 \%$ de probabilidade e n segue uma distribuição lognormal com 99\% de probabilidade.

Lassabatère et al. (2006) estudando três diferentes solos: um solo siltoso extremamente fino (Roujan); um solo estruturado com partículas maiores que $2 \mathrm{~mm}$ (Django Reinhardt) e um solo com aspectos texturais e estruturais intermediários aos dois primeiros solos (Chernobyl) obtiveram, respectivamente, os seguintes valores para $m, n$ e $\eta: 0,089 ; 2,20$ e 13,20 para Roujan; 0,327; 2,97 e 5,06 para Chernobyl; e 0,246; 2,65 e 6,07 para Django Reinhardt; já Souza et al. (2008a) obtiveram valores de 0,13, 2,31, 9,54 respectivamente, para $\mathrm{m}, \mathrm{n}, \eta \mathrm{em}$ um Neossolo Flúvico de textura areia franca e 0,$21 ; 2,54$ e 6,70 respectivamente para $m, n, \eta$ para um Neossolo Flúvico textura areia.

Mubarak et al. (2009) obtiveram, estudando as mudanças na estrutura do solo sob alta frequência de irrigação por gotejamento utilizando o método Beerkan, valores que variam entre 0,093 a 0,$095 ; 2,20$ a 2,21 e 12,52 a 12,93 para $m, n$ e $\eta$ respecticamente; os valores de $\mathrm{m}, \mathrm{n}$ e $\eta$ encontrados no presente trabalho estão próximos aos encontrados por esses autores.

Os semivariogramas dos parâmetros de forma $m, n$ e $\eta$ das duas áreas experimentais são apresentados na Figura 4; com exceção do valor $m$ da área sob pastagem, que foi ajustado ao modelo exponencial, todos foram ajustados pelo modelo esférico para as duas áreas.

O comportamento espacial dos parâmetros de forma das curvas $\theta(h)$ e $K(\theta)$ do solo sob pastagem e sob caatinga,
A.

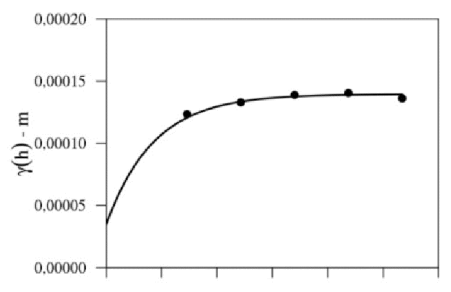

B.

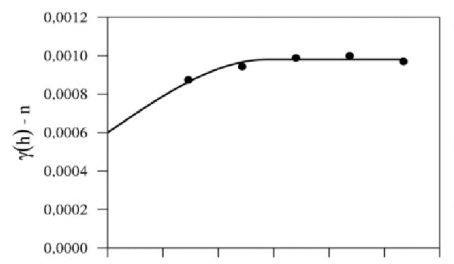

C.

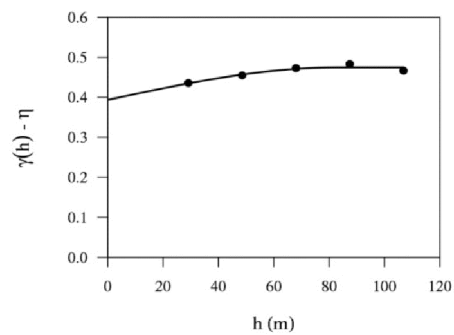

D.

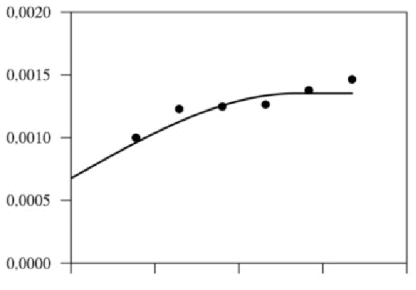

E.

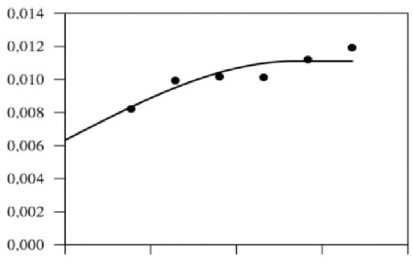

F.

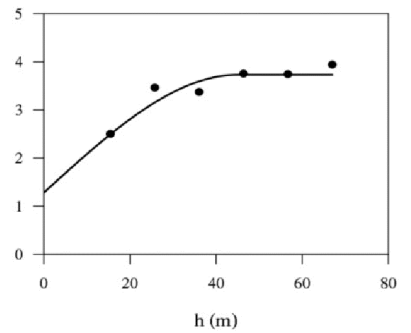

Figura 4. Semivariogramas experimental e teórico dos parâmetros de forma das curvas $\theta(h)$ e $K(\theta)(m, n$ e $\eta$ ) do solo sob pastagem (A, B e C) e sob caatinga (D, E e F)

Tabela 3. Estatística dos parâmetros de forma das curvas $\theta(h)$ e $K(\theta)(m, n$ e $\eta)$ do solo sob pastagem e sob caatinga

\begin{tabular}{|c|c|c|c|c|c|c|}
\hline \multirow{2}{*}{ Parâmetros estatísticos } & \multicolumn{3}{|c|}{ Pastagem } & \multicolumn{3}{|c|}{ Caatinga } \\
\hline & $\mathbf{m}$ & $n$ & $\eta$ & $\mathbf{m}$ & $\mathbf{n}$ & $\eta$ \\
\hline Média $(\mu)$ & 0,128 & 2,296 & 9,86 & 0,133 & $0,363(2,311)$ & 9,96 \\
\hline Mediana (Med) & 0,126 & 2,290 & 9,92 & 0,124 & $0,359(2,284)$ & 10,04 \\
\hline Variância $\left(\sigma^{2}\right)$ & 0,0001 & 0,0011 & 0,50 & 0,001 & $0,0001(0,001)$ & 3,38 \\
\hline Coef. de Variação (CV\%) & 9,499 & 1,418 & 7,178 & 26,17 & $4,95(4,27)$ & 18,47 \\
\hline Valor Máximo $\left(\mathrm{V}_{\text {máx }}\right)$ & 0,173 & 2,418 & 12,12 & 0,255 & $0,429(2,686)$ & 14,80 \\
\hline Valor Mínimo $\left(\mathrm{V}_{\min }\right)$ & 0,099 & 2,219 & 7,78 & 0,072 & $0,334(2,156)$ & 5,92 \\
\hline Número de observações (N) & 120 & 120 & 120 & 101 & 101 & 101 \\
\hline Kolmogorov - Smirnov (valor - $p$ ) & $0,217^{*}$ & $0,131^{*}$ & $0,798^{*}$ & 0,020 ** & $0,011^{* *}$ & $0,490^{*}$ \\
\hline
\end{tabular}

*Normal a $95 \%$ de probabilidade $(p>0,05)$; ${ }^{* *}$ Normal a $99 \%$ de probabilidade $(p>0,01)$ 
é apresentado na Figura 5. Observa-se uma disposição muito semelhante entre $\mathrm{m}$ e $\mathrm{n}$, além de um comportamento contrário para o parâmetro $\eta$ para as duas áreas analisadas, fato devido, provavelmente, ao formalismo matemático e à relação que envolve os parâmetros $m, n$ e $\eta$, em que $m$ e $n$ são diretamente proporcionais e esses, por sua vez, são inversamente proporcionais a $\eta$.

A estatística dos parâmetros hidrodinâmicos sorvidade (S) a condutividade hidráulica saturada $\left(\mathrm{K}_{\mathrm{s}}\right)$ e o potencial de entrada de $\operatorname{ar}\left(\mathrm{h}_{\mathrm{g}}\right)$ são apresentados na Tabela 4 . Os valores médios de $\mathrm{S}, \mathrm{K}_{\mathrm{s}}$ e $\mathrm{h}_{g}$ foram, respectivamente, $0,791 \mathrm{~mm} \mathrm{~s}^{-1 / 2}, 0,063 \mathrm{~mm}$ $\mathrm{s}^{-1} \mathrm{e}-20,67 \mathrm{~mm}$ para o solo sob pastagem e $0,939 \mathrm{~mm} \mathrm{~s}^{-1 / 2}$, $0,125 \mathrm{~mm} \mathrm{~s}^{-1} \mathrm{e}-17,67 \mathrm{~mm}$ respectivamente para $\mathrm{S}, \mathrm{K}_{\mathrm{s}}$ e $\mathrm{h}_{\mathrm{g}}$ do solo sob caatinga. Os valores médios de todos os parâmetros

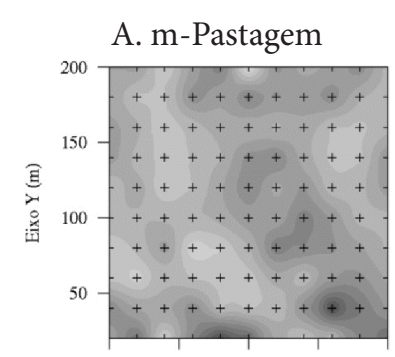

C. n-Pastagem

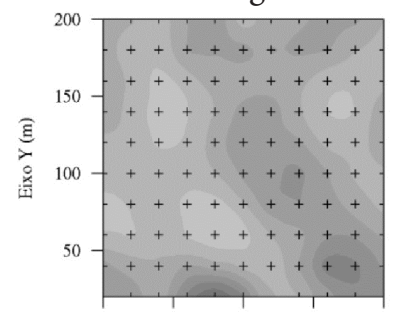

E. $\eta$-Pastagem

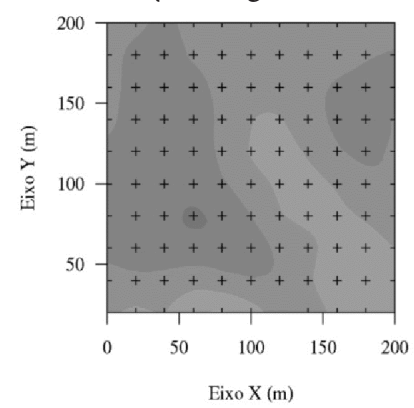

Figura 5. Mapa de isolinhas para os parâmetros de forma das curvas $\theta(h)$ e $K(\theta)$ (m, n e $\eta$ ) do solo sob pastagem e sob caatinga hidrodinâmicos da área de caatinga foram significativamente maiores que os da pastagem pelo teste $\mathrm{F}$ a $99 \%$ de probabilidade.

A sorvidade (S) traduz a capacidade do solo em absorver água por capilaridade e depende essencialmente da variação do teor volumétrico de água entre o início e o final da infiltração. De acordo com Souza et al. (2008a) este parâmetro varia para cada tipo de solo dependendo essencialmente da sua estrutura e da sua umidade inicial $\theta_{0}$.

Esperava-se, sem dúvida, que o solo da caatinga, o qual obteve menores valores de $\theta_{0}$ e $\rho_{s}$, apresentasse valores de $S$ maiores que no solo sob pastagem. De forma semelhante ocorre para a condutividade hidráulica do solo saturado $\left(\mathrm{K}_{\mathrm{s}}\right)$ que apresentou valores maiores para solo sob caatinga que sob pastagem, diferença que pode ser atribuída tanto à distribuição dos tamanhos das partículas da fração de areia fina como à densidade do solo nos dois usos, $1,39 \mathrm{~kg} \mathrm{dm}^{-3}$ para o solo sob pastagem e $1,30 \mathrm{~kg} \mathrm{dm}^{-3}$ para o solo sob caatinga.

Constata-se um efeito negativo da pastagem nos parâmetros hidrodinâmicos haja vista que, os valores médios de $\mathrm{K}_{\mathrm{s}}$ para o solo com caatinga foi quase o dobro do solo com pastagem, além de que a sorvidade também foi afetada pela pastagem a qual sofreu uma redução de cerca de $20 \%$ em média quando comparada com o solo sob caatinga.

Segundo Parente et al. (2010) um solo bem estruturado, menos compactado, pode apresentar valores de sorvidade e condutividade maiores e, devido ao conteúdo de matéria orgânica, consegue reter mais água.

Souza et al. (2007) observaram, avaliando o efeito do encrostamento superficial nas propriedades hidráulicas de um solo cultivado com mamona, que os valores da condutividade hidráulica e da sorvidade foram maiores para as superfícies sem crosta que para as superfícies com crosta, estando de acordo com as diferenças observadas nas lâminas infiltradas enquanto os valores de $\theta_{0}$ e de $\theta_{\mathrm{s}}$ foram menores para as superfícies sem crostas que para as superfícies com crostas.

Souza et al. (2008a) estimaram a sorvidade e a condutividade hidráulica com o programa BEST em três amostras de um Latossolo Amarelo e três amostras de um Neossolo Flúvico, e verificaram variação entre 0,49 e $2,48 \mathrm{~mm} \mathrm{~s}^{-1 / 2}$ para a sorvidade e entre 0,01 e $0,19 \mathrm{~mm} \mathrm{~s}^{-1}$ para a condutividade hidráulica saturada.

Segundo os limites de coeficiente de variação $(\mathrm{CV})$ todos os parâmetros hidrodinâmicos estudados apresentaram média variabilidade, com exceção do parâmetro $h_{g}$ do solo sob pastagem que obteve alta variabilidade.

Tabela 4. Estatística descritiva dos parâmetros hidrodinâmicos $\left(S, K_{s}\right.$ e $\left.h_{g}\right)$ do solo sob pastagem e sob caatinga

\begin{tabular}{|c|c|c|c|c|c|c|}
\hline \multirow{2}{*}{ Parâmetros estatísticos } & \multicolumn{3}{|c|}{ Pastagem } & \multicolumn{3}{|c|}{ Caatinga } \\
\hline & $S\left(\mathrm{~mm} \mathrm{~s}^{-1 / 2}\right)$ & $\mathrm{K}_{\mathrm{s}}\left(\mathrm{mm} \mathrm{s}^{-1}\right)$ & $\log \left|h_{g}\right|(m m)$ & $S\left(\mathrm{~mm} \mathrm{~s}^{-1 / 2}\right)$ & $\mathrm{K}_{\mathrm{s}}\left(\mathrm{mm} \mathrm{s}^{-1}\right)$ & $\log \left|\mathrm{h}_{\mathrm{g}}\right|(\mathrm{mm})$ \\
\hline Média $(\mu)$ & 0,791 & 0,063 & $1,175(-20,67)$ & 0,939 & 0,125 & $1,098(-17,67)$ \\
\hline Mediana (Med) & 0,745 & 0,052 & $1,339(-13,61)$ & 0,908 & 0,110 & $1,100(-13,82)$ \\
\hline Variância $\left(\sigma^{2}\right)$ & 0,088 & 0,002 & $0,107(518,63)$ & 0,137 & 0,008 & $0,120(127,86)$ \\
\hline Coef. de Variação (CV\%) & 37,58 & 62,45 & $27,81(-110,15)$ & 39,34 & 70,06 & $31,53(63,95)$ \\
\hline Valor Máximo $\left(\mathrm{V}_{\text {máx }}\right)$ & 1,998 & 0,288 & $2,301(-3,86)$ & 2,390 & 0,467 & $3,200(-6,486)$ \\
\hline Valor Mínimo $\left(\mathrm{V}_{\text {min }}\right)$ & 0,150 & 0,008 & $0,586(-200,00)$ & 0,2810 & 0,001 & $0,209(-71,23)$ \\
\hline Número de observações (N) & 120 & 120 & 120 & 101 & 101 & 101 \\
\hline Kolmogorov - Smirnov (valor-p) & $0,402^{*}$ & $0,027 * \star$ & $0,169 *$ & $0,500^{*}$ & $0,120^{*}$ & $0,190^{*}$ \\
\hline
\end{tabular}

Entre parênteses são apresentados os valores sem transformação; *Distribuição Normal a 95\% de probabilidade; *Distribuição Normal a $99 \%$ de probabilidade 
A distribuição segundo o teste Kolmogorov-Smirnov de todos os parâmetros hidrodinâmicos com exceção de $h_{\mathrm{g}}$ do solo sob pastagem, foi normal com $95 \%$ de probabilidade.

Os parâmetros $\mathrm{S}, \mathrm{K}_{\mathrm{s}} \mathrm{e} \mathrm{h}_{\mathrm{g}}$ do solo sob pastagem apresentaram dependência espacial moderada, segundo o critério estabelecido por Cambardella et al. (1994); já para o solo sob caatinga não foi detectada estrutura de dependência espacial para esses parâmetros (Figura 6). A ausência de dependência indica que a distribuição espacial do atributo na área de estudo é homogênea, aleatória ou que a malha amostral utilizada não possui pontos suficientes para detectar a dependência que, se existir, será manifestada a distâncias menores que o menor espaçamento entre amostras (Guimarães, 2004).

A Figura 7 apresenta os mapas de isolinhas para os parâmetros hidrodinâmicos $\mathrm{K}_{\mathrm{s}} \mathrm{e} \mathrm{h}_{\mathrm{g}}$ das duas áreas estudadas e de $S$ para a área de pastagem.

Observa-se que os mapas de $\mathrm{S}$ e $\mathrm{K}_{\mathrm{s}}$ apresentam comportamento semelhante podendo-se observar que as faixas de solo mais condutivas são as que também apresentam altos valores de sorvidade. De forma semelhante, Souza et al. (2008c) relataram que os mapas de S e lnKs para um Neossolo Flúvico sinalizam certa semelhança, em termos de padrão de variabilidade.

$\mathrm{Na}$ análise visual dos mapas de $\mathrm{S}$ e $\mathrm{K}_{s}$ (Figura 7) do solo sob pastagem observa-se pequena tendência de maiores valores na margem inferior direita e na margem superior ao centro;
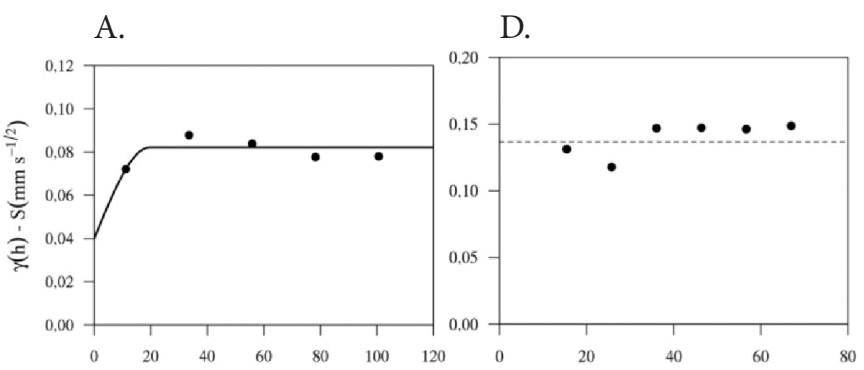

B.

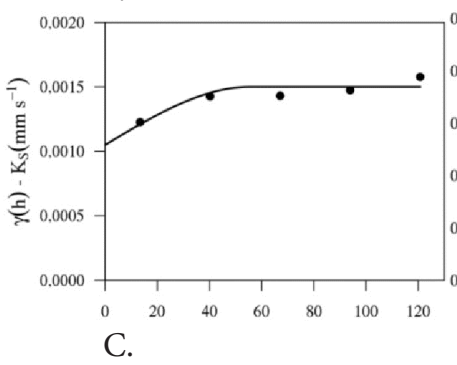

E.
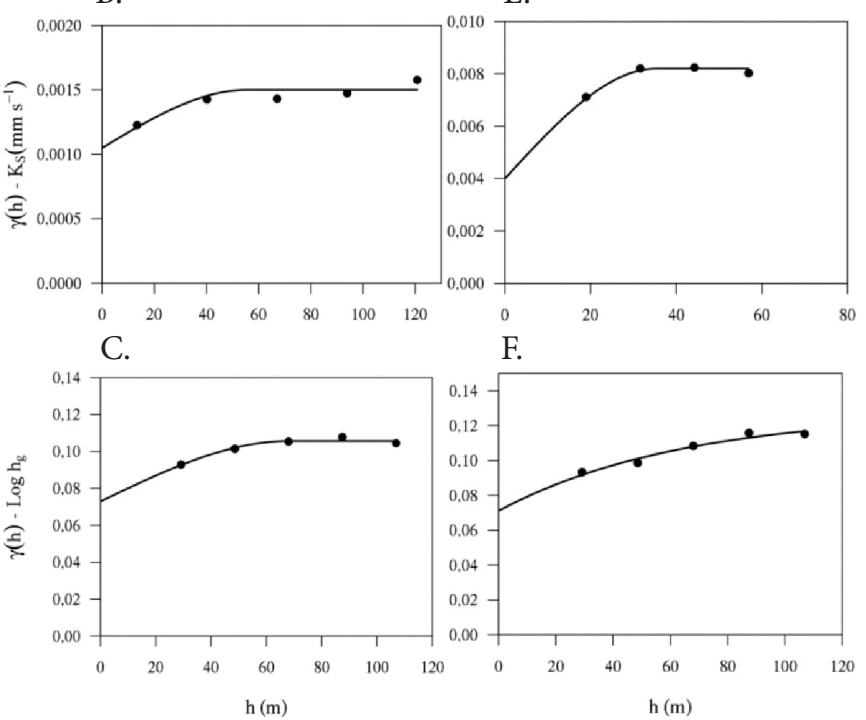

Figura 6. Semivariogramas experimental e teórico dos parâmetros hidrodinâmicos $\left(\mathrm{S}, \mathrm{K}_{\mathrm{s}}\right.$ e $\left.\log \mathrm{h}_{\mathrm{g}}\right)$ do solo sob pastagem (A, B e C) e sob caatinga (D, E e F)
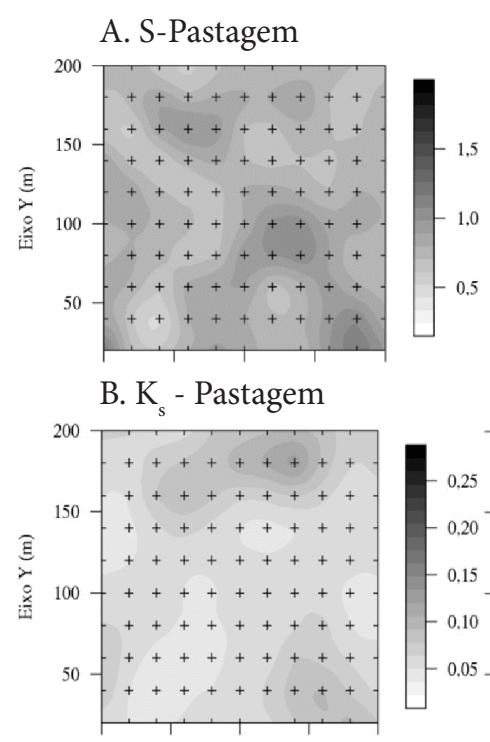

C. $\mathrm{K}_{\mathrm{s}}$ - Caatinga
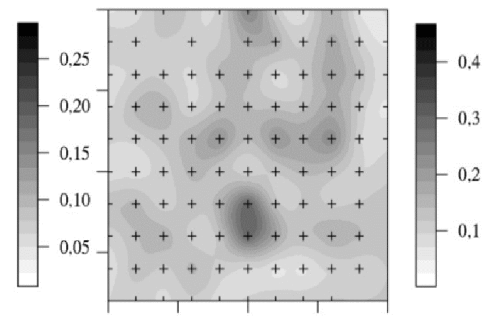

D. $\log \mathrm{h}_{\mathrm{g}}$ - Pastagem

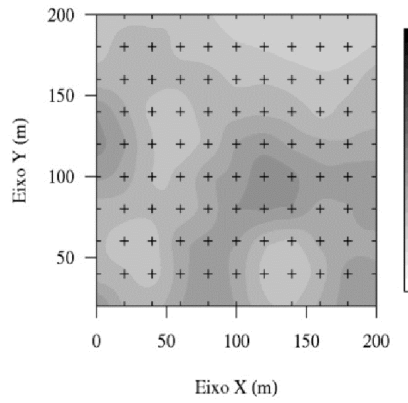

E. $\log \mathrm{h}_{\mathrm{g}}$ - Caatinga

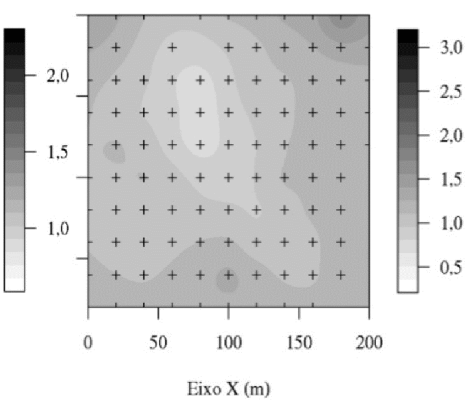

Figura 7. Mapa de isolinhas para os parâmetros hidrodinâmicos $\left(\mathrm{S}, \mathrm{K}_{\mathrm{s}}\right.$ e $\left.\operatorname{logh}_{\mathrm{g}}\right)$ do solo sob pastagem e sob caatinga

tal comportamento é pouco semelhante ao da disposição dos parâmetros $\mathrm{m}$ e n (Figura 5); para $\mathrm{o} \mathrm{h}_{\mathrm{g}}$ do solo sob pastagem nota-se um gradiente do centro às margens; essa distribuição se assemelha à distribuição do $\log \theta_{0}$ (Figura 3 ).

No solo de caatinga os maiores valores de $\mathrm{K}_{\mathrm{s}}$ têm uma pequena concentração do lado direito do mapa e os maiores valores de $\operatorname{lnh}_{\mathrm{g}}$, em termos absolutos, se concentram do centro para o lado esquerdo do mapa.

\section{Conclusões}

1. O manejo do solo com pastagem alterou significativamente todas as variáveis analisadas com destaque para a densidade do solo $\left(\rho_{\mathrm{s}}\right)$ e a condutividade hidráulica saturada $\left(\mathrm{K}_{\mathrm{s}}\right)$, que foram as mais afetadas pelo pisoteio dos animais.

2. Com exceção das $\rho_{\mathrm{s}}$ e $\theta_{\mathrm{s}}$, na pastagem, e da sorvidade, na caatinga, a análise geoestatística identificou a existência de estrutura de dependência espacial dos demais atributos estudados e permitiu seus mapeamentos utilizando-se técnicas da geoestatística e Krigagem.

\section{Agradecimentos}

À CAPES, pela bolsa de estudo; ao CNPq (processos 475094/2009-3; 305727/2009-5), à FACEPE (processos 
APQ-0077-5.01/09; APQ-1178-3.01/10) e à FINEP (processo 551922/2011-7) pelos recursos financeiros.

\section{Literatura Citada}

ABNT - Associação Brasileira de Normas Técnicas. NBR 7181 - Solo: análise granulométrica. Método de ensaio. Rio de Janeiro: ABNT, 1984. 13p.

Braud, I.; Condappa, D. de; Soria, J. M.; Haverkamp, R.; AnguloJaramillo, R.; Galle, S.; Vauclin, M. Use of scaled forms of the infiltration equation for the estimation of unsaturated soil hydraulic properties (the Beerkan method). European Journal of Soil Science, v.56, p.361-374, 2004.

Brooks, R. H.; Corey, A. T. Hydraulic properties of porous media. Hydrology. Paper, v.3, Fort Collins: Colorado State University, 1964.37p.

Burdine, N. T. Relative permeability calculation from size distribution data. America Institute Mining and Metallurgy Engineering, v.198, p.71-78, 1953.

Cambardella, C. A.; Moorman, T. B.; Novak, J. M.; Parkim, T. B.; Karlen, D. I.; Turco, R. F.; Koopka, A. E. Field-scale variability of soil properties in Central Iowa Soils. Soil Science Society of America Journal, v.58, p.1501-1511, 1994.

Carvalho, J. R. P.; Vieira, S. R.; Grego, C. R. Comparação de métodos para ajuste de modelos de semivariograma da precipitação pluvial anual. Revista Brasileira de Engenharia Agrícola e Ambiental, v.13, p.443-448, 2009.

Diggle, P. J.; Ribeiro Júnior, P. J. Model based geostatistics. New York: Springer, 2007. 230p.

Furtunato, O. M.; Montenegro, S. M. G. L.; Antonino, A. C. D.; Oliveira, L. M. M.; Souza, E. S.; Moura, A. E. S. S. Variabilidade Espacial de Atributos Físico-hídricos de Solos em uma Bacia Experimental no Estado de Pernambuco. Revista Brasileira de Recursos Hídricos, v.18, p.135-147, 2013.

Flores, J. P. C. Atributos físicos do solo e rendimento de soja em sistema plantio direto em integração lavoura pecuária com diferentes pressões de pastejo. Revista Brasileira de Ciência do Solo, v.31, p.771-780, 2007.

Guerra, P. A. L. G. Geoestatística operacional. Brasília: Ministério das Minas e Energia - Departamento Nacional da Produção Mineral, 1988. 145p.

Guimarães, E. C. Geoestatística básica e aplicada. Uberlândia: UFU, 2004. 76p.

Haverkamp, R.; Arrué, J. L.; Vandervaere, J. P.; Braud, I.; Boulet, G.; Laurent, J. P.; Taha, A.; Ross, P. J.; Angulo-Jaramillo, R. Hydrological and thermal behaviour of the vadose zone in the area of Barrax and Tomelloso (Spain): Experimental study, analysis and modeling. France: LTHE, 1996.

Lassabatère, L.; Angulo-Jaramillo, R.; Soria, J. M.; Cuenca, R.; Braud, I.; Haverkamp, R. Beerkan estimation of soil transfer parameters through infiltration experiments - BEST. Soil Science Society of American Journal, v.70, p.521-532, 2006.

Minasny, B.; Mcbratney, A. The efficiency of various approaches to obtaining estimates of soil hydraulic properties. Geoderma, v.107, p.55-70, 2002.
Mubarak, I.; Mailhol, J. C.; Angulo-Jaramillo, R.; Ruelle P.; Boivin, P.; Khaledian, M. Temporal variability in soil hydraulic properties under drip irrigation. Geoderma, v.150, p.158-165, 2009.

Oliveira, G. C.; Severiano, E. C.; Mello, C. R. Dinâmica da resistência à penetração de um Latossolo Vermelho da Microrregião de Goiânia, GO. Revista Brasileira de Engenharia Agrícola e Ambiental, v.11, p.265-270, 2007.

Parente, H. N.; Silva, D. S.; Andrade, A. P.; Souza, E. S.; Araújo, K. D.; Maia, M. O. Impacto do pisoteio caprino sobre atributos do solo em área de caatinga. Revista Brasileira de Saúde e Produção Animal, v.11, p.331-341, 2010.

R Development Core Team. R: A language and environment for statistical computing. Vienna: R Foundation for Statistical Computing, 2013.

Sales, L. E. O.; Carneiro, M. A. C.; Severiano, E. C.; Oliveira, G. C.; Ferreira, M. M. Qualidade física de neossolo quartzarênico submetido a diferentes sistemas de uso agrícola. Revista Ciência e Agrotecnologia, v.34, p.667-674, 2010.

Santos, J. C. B.; Souza Júnior, V. S.; Corrêa, M. M.; Ribeiro, M. R.; Almeida, M. C.; Borges, L. E. P. Caracterização de Neossolos Regolíticos da Região Semiárida do Estado de Pernambuco. Revista Brasileira Ciência do Solo, v.36, p.683-695, 2012.

Severiano, E. C.; Oliveira, G. C.; Curi, N.; Dias Júnior, M. S. Potencial de uso e qualidade estrutural de dois solos cultivados com cana-de-açúcar em Goianésia, GO. Revista Brasileira de Ciência do Solo, v.33, p.159-168, 2009.

Silva, A. J. N.; Cabeda, M. S. V. Compactação e compressibilidade do solo sob sistema de manejo e níveis de umidade. Revista Brasileira Ciência do Solo, v.30, p.921-930, 2006.

Souza, E. S.; Antonino, A. C. D.; Jaramillo, R. A.; Maciel Netto, A. Caracterização hidrodinâmica de solos: Aplicação do método Beerkan. Revista Brasileira de Engenharia Agrícola e Ambiental, v.12, p.128-135, $2008 \mathrm{a}$.

Souza, E. S.; Antonino, A. C. D.; Jaramillo, R. A.; Maciel Netto, A.; Montenegro, S. M. G. L.; Silva, E. B. Variabilidade espacial dos parâmetros hidrodinâmicos de duas parcelas agrícolas no estado da Paraíba. Revista Brasileira Ciência do Solo, v.32, p.1795-1804, 2008b.

Souza, E. S.; Antonino, A. C. D.; Lima, J. R. S.; Gouveia Neto, G. C.; Silva, J. M.; Silva, I. F. Efeito do encrostamento superficial nas propriedades hidráulicas de um solo cultivado com mamona. Revista Brasileira de Ciências Agrárias, v.2, p.69-74, 2007.

Souza, E. S.; Antonino, A. C. D.; Montenegro, S. M. G. L.; Lima, J. R. S.; Ângulo-Jaramillo, R. Mapeamento da probabilidade de ocorrência de classes da sorvidade e da condutividade hidráulica saturada de um Neossolo Flúvico. Revista Brasileira de Ciências Agrárias, v.3, p.164-172, 2008c.

van Genuchten, M.Th. van. A closed-form equation for predicting the hydraulic conductivity of unsaturated soils. Soil Science Society of America Journal, v.44, p.892-898, 1980. Vieira, S. R.; Carvalho, J. R. P.; González, A. P. Jack knifing for semivariogram validation. Bragantia, v.69, p.97-105, 2010.

Warrick, A. W.; Nielsen, D. R. Spatial variability of soil physical properties in the field. In: Hillel, D. (Ed.) Applications of Soil Physics. New York: Academic Press, 1980. p.319-344. 